\title{
Occurrence of the Redifferentiation-Akin Phenomenon on ${ }^{68}$ Ga-DOTATATE PET/CT After CAPTEM Chemotherapy in Metastatic Neuroendocrine Tumors with Intermediate MIB1 Index: What Could Be the Molecular Explanation?
}

Priyanka Sharma and Sandip Basu

Radiation Medicine Centre, Bhabha Atomic Research Centre, Tata Memorial Centre Annexe, and Homi Bhabha National Institute, Mumbai, India

In this report, we present the ${ }^{68} \mathrm{Ga}$-DOTATATE PET/CT images of a patient with a metastatic neuroendocrine tumor with an intermediate-grade/Ki-67 index who had minimal somatostatin receptor positivity on baseline ${ }^{68} \mathrm{Ga}$-DOTATATE PET/CT but high-grade positivity on somatostatin receptor imaging (Krenning score, III-IV) on follow-up scans after chemotherapy. This redifferentiation-akin phenomenon in intermediate-grade neuroendocrine tumors after chemotherapy can have potential implications for the feasibility of peptide receptor radionuclide therapy in this group of patients in the later phase of their disease course. Key Words: ${ }^{68} \mathrm{Ga}$-DOTATATE; PET/CT; neuroendocrine tumor; peptide receptor radionuclide therapy

J Nucl Med Technol 2020; 48:290-291

DOI: 10.2967/jnmt.120.241539

\section{$\mathbf{P}$} eptide receptor radionuclide therapy with ${ }^{177} \mathrm{Lu}-$ DOTATATE has recently emerged as a front-runner therapy for well-differentiated and intermediate-grade neuroendocrine tumors (NETs). Its favorable status can be attributed to its better patient tolerability, its convenient treatment schedule and cycle interval, and its excellent efficacy in alleviating symptoms. However, some intermediate-grade NETs show minimal somatostatin receptor (SSTR) positivity, thus rendering them unsuitable for peptide receptor radionuclide therapy. In our set-up, a few such cases that have been followed up with PET imaging after chemotherapy (e.g., capecitabine-temozolomide [CAPTEM] therapy) have shown enhancement of tracer uptake with high-grade positivity on SSTR imaging (1). This small subset of patients may be potential candidates for peptide receptor radionuclide therapy.

Received Jan. 1, 2020; revision accepted Feb. 15, 2020.

For correspondence or reprints contact: Sandip Basu, Radiation Medicine Centre, Bhabha Atomic Research Centre, Tata Memorial Hospital Annexe, Jerbai Wadia Rd., Parel, Mumbai, Maharashtra, India, 400012.

E-mail: drsanb@yahoo.com

Published online Feb. 28, 2020

COPYRIGHT (c) 2020 by the Society of Nuclear Medicine and Molecular Imaging.

\section{CASE REPORT}

A 58-y-old woman diagnosed with metastatic grade 2 gastric NET (histopathology demonstrating well-differentiated NET, positive for AE1/AE2/AE3, synaptophysin, and chromogranin and negative for C-kit and DOG1, with an MIB1 index of 7\%$8 \%$ ) was referred to investigate the feasibility of peptide receptor radionuclide therapy. The contrast-enhanced CT scan demonstrated a soft-tissue lesion probably arising from an exophytic soft-tissue mass in the stomach and abutting the left lobe of the liver. There were also multiple liver lesions, the largest being in the right lobe, with areas of necrosis. The ${ }^{68} \mathrm{Ga}$-DOTATATE PET/CT images showed low-grade SSTR expression in the soft-tissue mass (measuring $10 \times 6 \times 5.3 \mathrm{~cm}$; maximum

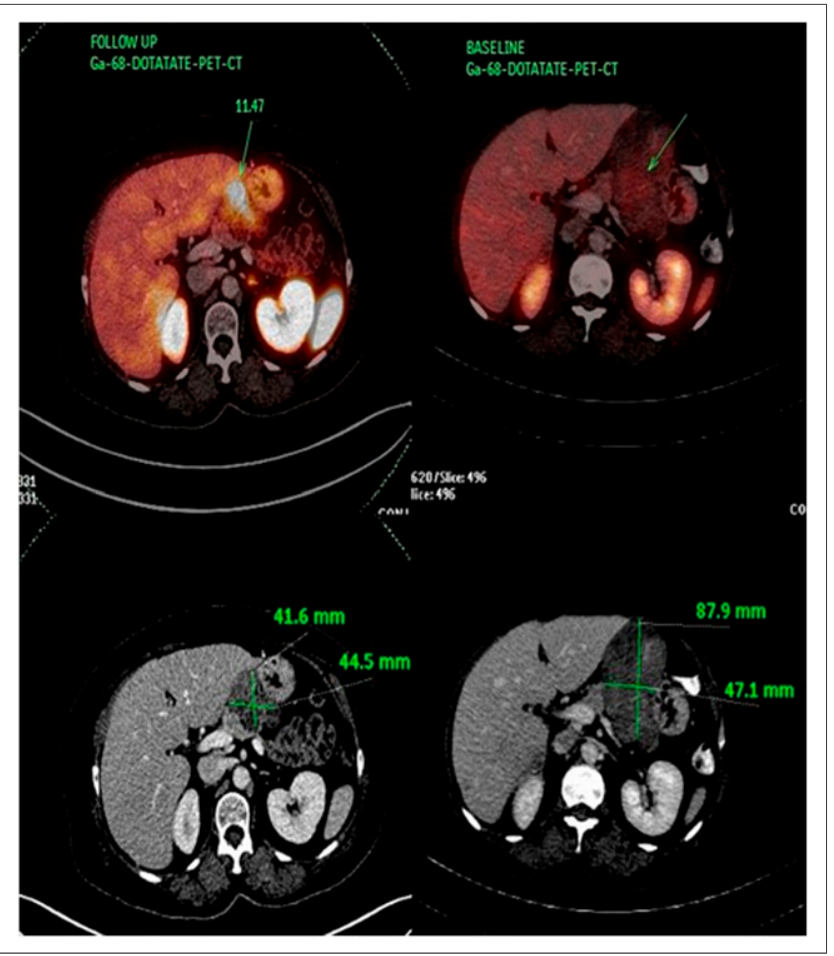

FIGURE 1. Tracer uptake $\left(S U V_{\max }, 11.47\right)$ in primary gastric mass on follow-up axial ${ }^{68}$ Ga-DOTATATE PET/CT (top) and CT (bottom) images (left) compared with baseline images (right). 


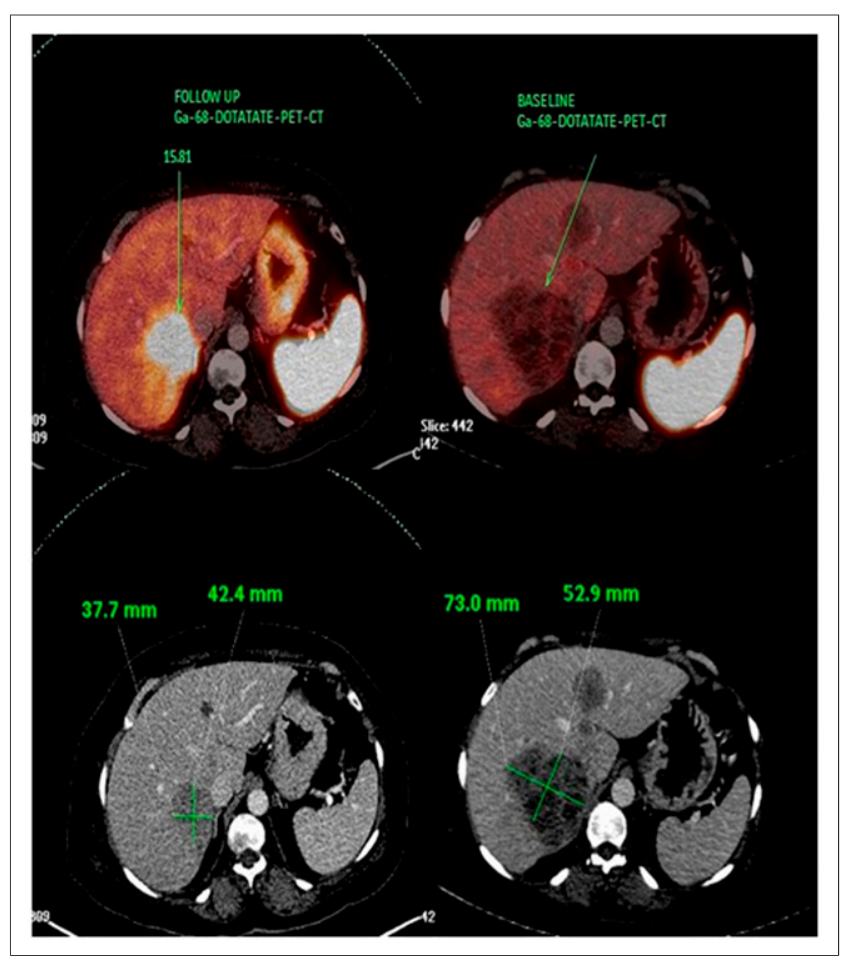

FIGURE 2. Krenning score 3 uptake in segment VII and VIII liver lesions on follow-up axial ${ }^{68} \mathrm{Ga}-\mathrm{DOTATATE}$ PET/CT (top) and CT (bottom) images (left) compared with baseline images (right). Lesions are seen to be smaller on follow-up.

SUV, 9.09), involving the lesser curvature of the stomach, consistent with known primary and minimal uptake in the metastatic liver lesions (Krenning score $\leq 1$ ), with the largest lesion being in segment VII/VIII (Fig. 1). In view of the low-to-minimal uptake on SSTR-based PET imaging and the MIB1 index of 7\%-8\%, CAPTEM chemotherapy was considered for this patient. She showed a clinical response in terms of abdominal pain reduction. After 6 cycles of CAPTEM, follow-up ${ }^{68} \mathrm{Ga}$ DOTATATE PET/CT was performed (Figs. 1-3) and showed increased SSTR expression (Krenning score, 3) at the gastric mass and the liver lesions, with a significant reduction in the size of the gastric mass and the liver lesions.

\section{DISCUSSION}

Uptake on ${ }^{68} \mathrm{Ga}$-DOTATATE PET/CT images is an important prerequisite for choosing peptide receptor radionuclide therapy for metastatic NETs. The Krenning score is used to grade the intensity of the NET uptake on SSTR-based imaging (2); typically, peptide receptor radionuclide therapy is considered when the Krenning score is greater than 2. CAPTEM-based chemotherapy is used successfully in patients with intermediate-grade NETs, especially in cases with poor or minimal SSTR expression.

To our knowledge, the existing literature is limited on the topic of chemotherapy-induced enhanced uptake on SSTR-based PET imaging, with only a single communication being found $(l)$. This redifferentiation-akin phenomenon in the parlance of NET is presently unexplained, but we have observed it in the context of intermediate-grade NETs. Basu et al. (1) cited 3 example cases

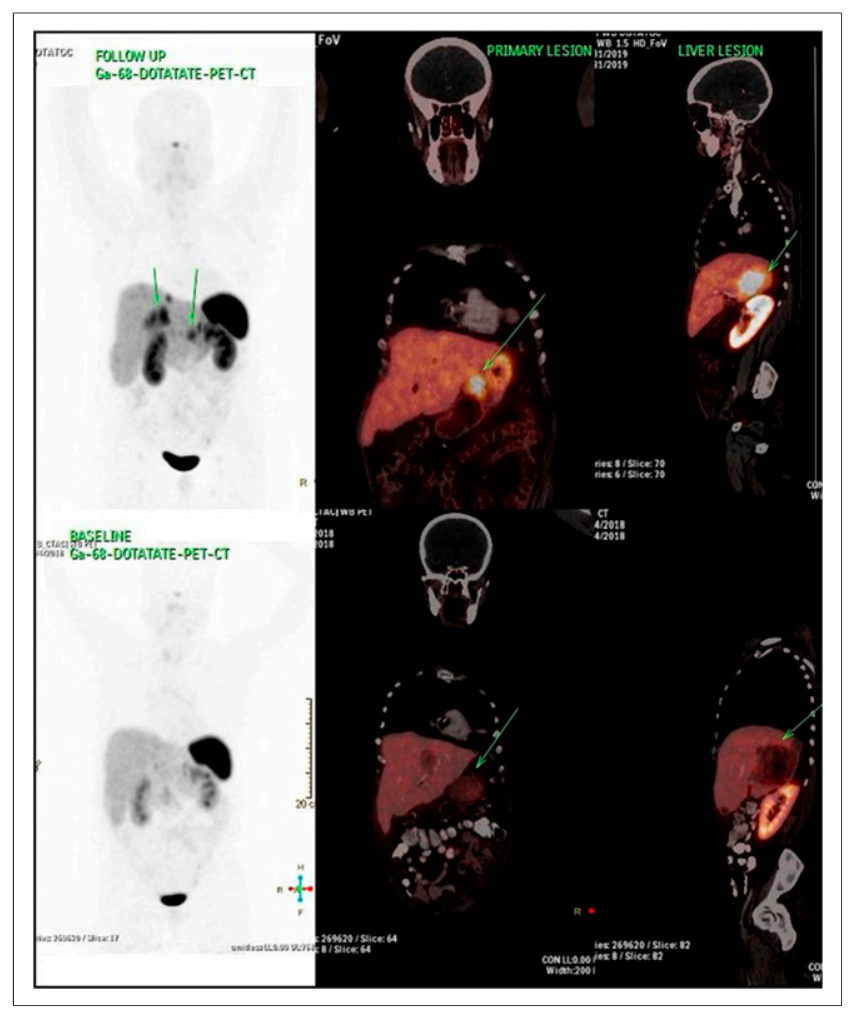

FIGURE 3. Baseline (bottom) and follow-up (top) maximumintensity projection PET images (left) and coronal (middle) and sagittal (right) ${ }^{68} \mathrm{Ga}-\mathrm{DOTATATE}$ PET/CT images. Tracer uptake is seen to be enhanced on follow-up.

in which patients with intermediate-grade progressive metastatic NET presented with minimal uptake on an initial work-up with ${ }^{68} \mathrm{Ga}$-DOTATATE PET/CT. As a result, these patients were treated with CAPTEM-based chemotherapy and showed intense uptake on subsequent ${ }^{68} \mathrm{Ga}$-DOTATATE PET/CT scans, making them eligible for peptide receptor radionuclide therapy at a later period. Although we noted enhanced ${ }^{68} \mathrm{Ga}$-DOTATATE uptake in a limited population, it is premature to state that SSTR increases in every NET patient who has been treated with CAPTEM. This possibility should be clarified in a prospective setting.

The present case upholds a similar observation and emphasizes the need for an appropriate clinical protocol to benefit such a subset of patients who might later benefit from peptide receptor radionuclide therapy if follow-up scans show increased SSTR expression.

\section{CONCLUSION}

The redifferentiation-akin phenomenon in intermediate-grade neuroendocrine tumors after chemotherapy can have potential implications for the feasibility of peptide receptor radionuclide therapy in this group of patients in the later phase of their disease course.

\section{REFERENCES}

1. Basu S, Ostwal V. Observation on enhanced avidity on somatostatin receptor targeted ${ }^{68} \mathrm{Ga}$-DOTATATE PET-CT following therapy with everolimus and capecitabine-temozolamide: is redifferentiation akin phenomenon a reality in neuroendocrine tumors? Nucl Med Commun. 2016;37:669-671.

2. Kwekkeboom DJ, Krenning EP. Somatostatin receptor imaging. Semin Nucl Med. 2002;32:84-91. 